\title{
Limits to the attentional boost effect: the moderating influence of orthographic distinctiveness
}

\author{
Pietro Spataro • Neil W. Mulligan • Clelia Rossi-Arnaud
}

Published online: 21 November 2014

(C) Psychonomic Society, Inc. 2014

\begin{abstract}
The Attentional Boost Effect (ABE) refers to the counter-intuitive finding that the detection of infrequent targets in a divided-attention (DA) condition enhances memory of images co-occurring with targets (as compared with images co-occurring with distractors; Swallow \& Jiang Cognition, $115,118-132,2010)$. Previous studies have shown that the ABE also applies to verbal materials (words; Spataro, Mulligan, \& Rossi-Arnaud Journal of Experimental Psychology: Learning, Memory, and Cognition, 39, 1223$1231,2013)$ and documented an important moderating factor, word frequency - the ABE was robust for high-frequency words, but small or non-significant for low-frequency words (Mulligan, Spataro, \& Picklesimer Journal of Experimental Psychology: Learning, Memory, and Cognition, 40, 10491063, 2014). The present experiment tested the predictions of the early-phase-elevated-attention hypothesis of the ABE by manipulating the orthographic distinctiveness of the to-beremembered words. Results revealed that the $\mathrm{ABE}$ was significant for low-frequency words with common orthographic features, but not for low-frequency words with rare orthographic features. As a consequence, the orthographic distinctiveness effect (ODE) was eliminated in the DA condition. These findings are in line with the proposal that the ABErelated attentional enhancement occurs during an early phase of stimulus perception and comprehension, as well as with the proposal that the ODE is mediated by high-level, attention demanding comparative processes.
\end{abstract}

P. Spataro $(\bowtie) \cdot$ C. Rossi-Arnaud

Department of Psychology, University of Rome "Sapienza", Via dei

Marsi 78, 00185 Rome, Italy

e-mail: pietro.spataro@uniroma1.it

N. W. Mulligan

University of North Carolina, Chapel Hill, USA
Keywords Memory and attention · Recognition · Word recognition

\section{Introduction}

Divided attention (DA) at encoding typically reduces memory performance in explicit tasks (Mulligan, 2008), as well as in some, but not all, implicit tasks (Spataro, Cestari, \& RossiArnaud, 2011). However, under specific circumstances, the detection of infrequent targets in a DA condition can enhance memory of background scenes (Swallow \& Jiang, 2010, 2013). Participants in these experiments encoded a long sequence of images, while simultaneously monitoring the colour of a small square located at the centre of each image. In the DA condition, they were to memorize the scenes and press the spacebar of the computer whenever they detected occasional white squares (targets) among more frequent black squares (distractors). In a later four-choice recognition task, Swallow and Jiang (2010) found higher hit rates for scenes encoded with targets than for scenes encoded with distractors, the attentional boost effect (ABE). No memory enhancement was observed in the full-attention (FA) condition, when participants ignored the squares. Importantly, Swallow and Jiang (2010) showed that the ABE reflects a trade-off between an 'attentional boost' process (i.e., memory for scenes accompanied by target squares in the DA condition was enhanced up to the same levels achieved in the FA condition) and an 'attentional competition' process (i.e., memory for scenes accompanied by distractor squares was worse in the DA than in the FA condition), the latter reflecting the usual negative effect of DA on explicit memory encoding.

In two previous studies, Spataro, Rossi-Arnaud and Mulligan (2013) and Mulligan, Spataro and Picklesimer (2014) demonstrated that the ABE also affects verbal explicit and implicit memory. In the DA condition, participants read 
aloud a series of words and concurrently monitored the colour of a small circle placed below each word, in order to detect infrequent red circles (targets) among more frequent green circles (distractors). In the first experiment reported by Spataro et al. (2013), the ABE was replicated using a fourchoice recognition task: memory for words encoded with targets in the DA condition was enhanced up to the same levels of the FA condition (attentional boost); in contrast, memory for words encoded with distractors was significantly reduced in the DA than in the FA condition (attentional competition). Mulligan et al. (2014) confirmed and extended these findings, but also documented an important moderating factor: the verbal $\mathrm{ABE}$ in recognition and free recall was robust for high-frequency words, but small or nonsignificant for low-frequency words. The authors interpreted this result to comport with the early-phase-elevated-attention account of word frequency, which proposes that, compared to high-frequency items, low-frequency words attract more attention during an early phase of encoding comprising stimulus perception and comprehension (Criss \& Malmberg, 2008; Malmberg \& Nelson, 2003). Because of their unusual properties, a larger amount of attention resources would be devoted to the elaboration of low-frequency words in these relatively automatic processing stages, resulting in a greater number of features of these words and the circumstances of their occurrence being remembered (Malmberg \& Nelson, 2003). According to Mulligan et al. (2014), the differential encoding accruing to target-related words in the ABE paradigm would also arise early at encoding, rendering this attentional manipulation largely redundant with the increased processing naturally occurring for low-frequency words. As a consequence, the transient attentional enhancements occurring as a consequence of target detection in the ABE paradigm should have minimal effects on the encoding of low-frequency words, because they are already subject to heightened attention.

The argument of the close relationship between the ABE and the early-phase-elevated-attention account of recognition memory has been examined in more depth by Mulligan and Spataro (in press). Building on previous research indicating that the advantage of low-frequency words (over highfrequency words) is robust at study times of $1 \mathrm{~s}$ per word and does not increase at longer study times (e.g., Hirshman \& Palij, 1992; Malmberg \& Nelson, 2003), these authors showed that a similar pattern of results also occurred for the ABE: the advantage of words encoded with targets (over words encoded with distractors) was significant at a short study duration of $700 \mathrm{~ms}$ and did not increase at study durations of 1500 and $4000 \mathrm{~ms}$. Such a result bolsters the hypothesis that the attentional boost of target-aligned words is based on early-phase processes of stimulus identification and comprehension, and does not involve late-phase controlled rehearsal processes, like interpretive elaboration and mental imagery (Hirshman, Trembath, \& Mulligan, 1994; MacLeod \& Masson, 1997).
Two other predictions, also derived from the early-phaseelevated-attention explanation of the ABE, were borne out by Mulligan and Spataro (in press). First, recognition accuracy in the DA condition reached the FA levels at a study duration of $700 \mathrm{~ms}$, whereas it fell significantly below the FA levels at a study duration of $4000 \mathrm{~ms}$, consistent with the claim that, when study time is sufficiently long, the negative effects of DA on late-phase controlled processes may exceed the ABErelated enhancement on early-phase processes. Conversely, when study time was reduced to $400 \mathrm{~ms}$, words presented with targets in the DA condition were recognized at higher rates than words presented in the FA condition, demonstrating that the $\mathrm{ABE}$ can produce an absolute facilitation when the contribution of late-phase processes is severely limited.

These results and theoretical analyses suggest broader implications about the relationship between the ABE and distinctiveness, as detailed below. These implications are best investigated by using a direct manipulation of distinctiveness known to impact memory, in the present case, orthographic distinctiveness. The orthographic distinctiveness effect (ODE) refers to the finding that words with rare letter combinations (i.e., unusual visual patterns) are remembered better than words with common letter combinations (Hunt \& Elliot, 1980). Interestingly, orthographic distinctiveness is one (but not the only) factor contributing to the memory advantage of low-frequency words (Malmberg, Steyvers, Stephens, \& Shiffrin, 2002). Although not explicitly framed in terms of the elevated-attention hypothesis, there is evidence suggesting that the ODE, like the $\mathrm{ABE}$ and the low-frequency-word advantage, depends on early-phase encoding processes. Gounden and Nicolas (2012a), in particular, showed that the magnitude of the ODE did not increase when the time allotted for processing words was manipulated $(250,500,1000$ or $3000 \mathrm{~ms}$ ). Consequently, we expect the attentional facilitation produced by target detection in the $\mathrm{ABE}$ paradigm to be redundant with the early processing advantage enjoyed by orthographically distinctive words (Mulligan et al., 2014).

More specifically, our prediction follows from the notion that the effects of distinctiveness are not absolute; instead, they presuppose the organization of the encoded information along common dimensions, since between-items differences are computed only in the context of similarity (Hunt \& Elliot, 1980; Smith \& Hunt, 2000). When high- and low-frequency words are presented together in the same study list (as it was the case in the experiments reported by Mulligan et al., 2014), the unusual features of low-frequency items become strongly distinctive, because participants have the possibility to evaluate the discrepancy with respect to the background of common features that make up high-frequency items (Malmberg \& Nelson, 2003; Malmberg et al., 2002); in this condition, low-frequency words attract more attentional resources, compared to high-frequency words, and the encoded differences have strong diagnostic value at the time of retrieval (Guttentag 
\& Carroll, 1997; MacLeod \& Kampe, 1996). However, iflowfrequency words are encoded in the context of other items having similar frequency but higher orthographic distinctiveness, then more attention should be devoted to the encoding of low-frequency orthographically distinctive (LF-OD) words, resulting in a less accurate processing of low-frequency orthographically common (LF-OC) words. Coupled with previous evidence indicating that the ABE produces little benefits to the encoding of stimuli which are already subject to heightened attention during the study phase (Mulligan et al., 2014), this hypothesis led us to expect that a significant boost-related facilitation should occur for LF-OC words but not for LF-OD words. Evaluation of this prediction dictates that orthographic distinctiveness is varied while (low) word frequency is held constant.

A second, related prediction stems from consideration of the relative nature of the $\mathrm{ABE}$, which, as mentioned above, reflects the combination between an attentional boost process (i.e., memory for words encoded with targets in the DA condition is raised to the FA levels) and an attentional competition process (i.e., memory for words encoded with distractors in the DA condition is reduced below the FA levels) (Mulligan et al., 2014; Spataro et al., 2013). In the present study, this pattern should be replicated on LF-OC words. However, for LF-OD words, no memory facilitation was expected for items encoded with targets in the DA condition. As a consequence, we predicted that performance for target-associated LF-OD words in the DA condition should not exceed the levels observed for distractor-associated LFOD words, and that accuracy in both of these conditions should be significantly lower than the FA levels. In other words, the effects of DA should be more deleterious for LFOD than for LF-OC words encoded with targets.

\section{Method}

Participants Thirty-four students of the University Sapienza of Rome (22 females; age: $M=25.7$ years) participated, 14 in the FA condition and 20 in the DA condition.

Design and materials The experiment used a $2 \times 2 \times 2$ mixed factorial design, in which Item Type (LF-OD vs. LF-OC words) and Trial Type (words encoded with targets vs. distractors) were manipulated within participants and Attention Condition (FA vs. DA) was manipulated between participants.

Sixty critical words were selected from the CoLFIS Vocabulary (http://linguistica.sns.it/CoLFIS/Home.htm). Thirty words had unusual letter combinations for Italian (e.g. , "ketchup") and thirty words had common letter combinations (e.g., "marmotta"), as assessed by computing mean bigram frequencies - that is, the averaged frequencies of all two-letter combinations in the word. This measure of orthographic distinctiveness was estimated from the database PhonItalia, which provides the frequency of 477 bigrams, as a function of their position in the word (http://www.psy. plymouth.ac.uk/research/jgoslin/phonitalia). After a log transformation, the resulting bigram frequencies were 3.63 for LF-OC words and 3.30 for LF-OD words, a significant by-item difference $[t(58)=-5.60, p<0.001]$. The two lists of critical words were otherwise equated as closely as possible on: (1) adult written frequency, as estimated with the CoLFIS vocabulary $[M(\mathrm{LF}-\mathrm{OD})=12.3$ vs. $M(\mathrm{LF}-\mathrm{OC})=13.4 ; t(58)=$ $0.05, p=0.096]$; and (2) length in letters $[M(\mathrm{LF}-\mathrm{OD})=7.4 \mathrm{vs}$. $M(\mathrm{LF}-\mathrm{OC})=7.3 ; t(58)=0.33, p=0.74]$. From this original set, three sub-lists of 10 LF-OD words and three sub-lists of 10 LF-OC words were created, for the purposes of counterbalancing. Finally, 58 non-critical words were selected to be used as practice or filler items during the encoding phase.

Procedure The experiment consisted of three consecutive phases. At encoding, participants were presented with 78 words (18 practice and 60 critical items), at a rate of $500 \mathrm{~ms}$ per word. On each trial, one word and one square (red or green) appeared simultaneously at the centre of the screen for $100 \mathrm{~ms}$, with a vertical distance of $1 \mathrm{~cm}$ between them, after which only the word remained visible for an additional $400 \mathrm{~ms}$. The study list was organized into 26 blocks of 3 words, with no interruption between successive trials. Half of the blocks were composed of LF-OD words and the other half of LF-OC words. Critical words encoded with red squares (target words) were always placed in the second position, whereas critical words encoded with green squares (distractor words) were randomly located either in the first or in the third position of each block. All other words in the blocks (non-critical) were associated with green squares. To reduce the regularity of target presentation, one to three filler words (encoded with green squares) were randomly interspersed between two consecutive blocks. In the DA condition, participants were told to read aloud and study each word while simultaneously pressing the spacebar of the computer whenever they detected a red square. In the FA condition, participants were told to ignore the squares and focus only on the words.

The encoding phase was followed by a 15-min interval, in which participants completed a series of distractor tasks. Lastly, a paper-and-pencil yes/no recognition task was administered. This included 40 studied items (10 LF-OD words encoded with targets, 10 LF-OD words encoded with distractors, 10 LF-OC words encoded with targets and 10 LF-OC words encoded with distractors) and 20 unstudied words (10 LF-OD and 10 LF-OC). Participants were instructed to mark the words they remembered from the encoding phase. 


\section{Results}

During the DA condition, participants correctly detected $99.4 \%$ of the target squares (mean RT $=372 \mathrm{~ms}$ ). Figure 1 (upper panel) illustrates corrected recognition scores (hits false alarms) for LF-OD and LF-OC words in the FA and DA conditions, and Table 1 (upper panel) reports hits and false alarms.

A mixed 2 (Item Type: LF-OD vs. LF-OC words) $\times 2$ (Trial Type: words encoded with target vs. distractor squares) $\times 2$ (Attention Condition: FA vs. DA) ANOVA revealed significant main effects of Item Type $[F(1,32)=15.26$, MSE $=$ 0.059, $\left.p<0.001, \eta_{p}^{2}=0.32\right]$ and Attention Condition $[F(1$, $\left.32)=4.64, \mathrm{MSE}=0.117, p<0.05, \eta_{p}^{2}=0.13\right]$, indicating that recognition memory was significantly better for LF-OD than for LF-OC words ( $M=0.58$ vs. $M=0.41$; i.e., the ODE was replicated), and better in the FA than in the DA condition $(M=$ 0.56 vs. $M=0.43)$. The main effect of Trial Type was marginally significant $\left[F(1,32)=3.44, \mathrm{MSE}=0.014, p=0.073, \eta_{p}^{2}=\right.$ 0.09], suggesting that performance tended to be higher for words encoded with targets than for words encoded with distractors $(M=0.52$ vs. $M=0.48)$. Crucially for the present purposes, the three-way interaction between all factors reached the significance level $[F(1,32)=4.95, \mathrm{MSE}=0.018, p<0.05$, $\left.\eta_{p}^{2}=0.13\right]$. A follow-up analysis of simple effects, using the Bonferroni adjustment, showed that:

a) in the DA condition, the ABE was significant for LF-OC words $\left[F(1,32)=7.59, p \leq 0.01, \eta_{p}^{2}=0.19\right]$, with recognition being better for words encoded with targets than for words encoded with distractors $(M=0.44$ vs. $M=$ 0.32), whereas there was no hint of an ABE for LF-OD words $[F(1,32)=0.52, p=0.48$; see Fig. 1$]$;

b) the interfering effect of DA was significant for LF-OC words encoded with distractors $[F(1,32)=5.58, \mathrm{MSE}=$ $\left.0.026, p<0.05, \eta_{p}^{2}=0.15\right]$ and for LF-OD words encoded with both targets and distractors $[F(1,32)>5.24, p<$ $\left.0.05, \eta_{p}^{2}>0.12\right]$ c) the ODE was eliminated for words encoded with targets in the DA condition $[M($ distinct $)=0.50$ vs. $M($ common $)=$ 0.48: $F(1,32)=0.31, p=0.57]$, whereas it was significant in all other conditions $[F(1,32)>6.78$, $\left.p \leq 0.01, \eta_{p}^{2}>0.18\right]$.

\section{Discussion}

The primary aim of the present study was to investigate the relationship between the $\mathrm{ABE}$ and the ODE; i.e., to determine whether these two effects can be accounted for by transient increases in the levels of attention during the early phases of memory encoding (Malmberg \& Nelson, 2003; Mulligan et al., 2014; Mulligan \& Spataro, in press). For this purpose, we tested whether target detection in the DA condition of the ABE paradigm could enhance memory of co-occurring lowfrequency words with common or distinctive orthographic properties. In line with our predictions, the results showed that a significant ABE occurred for LF-OC words but not for LF-OD words. Such a finding is consistent with the earlyphase-elevated-attention account of the ABE (Mulligan et al., 2014), as well as with the hypothesis that, in the context of mixed study lists, the encoding of orthographically distinctive words attracts more attentional resources, compared to the encoding of orthographically common words (Hunt \& Elliot, 1980). As discussed above, Mulligan et al. (2014) found that the $\mathrm{ABE}$ in recognition and free recall was robust for highfrequency words, but small or non-significant for lowfrequency words. They argued that target detection had a negligible influence on low-frequency words because their unusual properties resulted in a greater amount of attention being paid to them during the early phases of encoding (Criss \& Malmberg, 2008; Malmberg \& Nelson, 2003). The present study extends this conclusion, by showing that the ABE does not enhance the recognition of LF-OD words, when they are encoded in the context of other blocks containing LF-OC

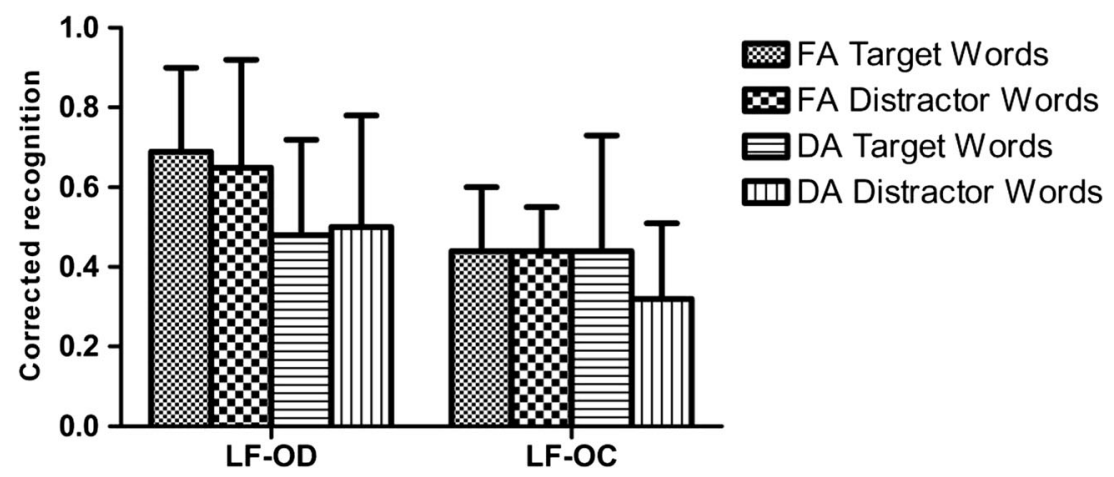

Fig. 1 Mean corrected recognition scores, as a function of Item Type (LF-OD vs. LF-OC words), Trial Type (words encoded with target vs. distractor squares) and Attention Condition (FA vs. DA). Bars represent

standard deviations. Note. LF-OD: low-frequency orthographically distinct words; LF-OC: low-frequency orthographically common words; FA: full attention; DA: divided attention 
Table 1 Mean correct hits and false alarms as a function of Item Type (LF-OD vs. LF-OC words), Trial Type (words encoded with target vs. distractor squares) and Attention Condition (Full vs. Divided)

\begin{tabular}{|c|c|c|c|c|}
\hline \multirow[t]{2}{*}{ Type of Words } & \multicolumn{2}{|c|}{ LF-OD Words } & \multicolumn{2}{|l|}{ LF-OC Words } \\
\hline & Correct Hits & False Alarms & Correct Hits & False Alarms \\
\hline \multicolumn{5}{|l|}{ Full Attention } \\
\hline Words encoded with targets & $0.90(0.07)$ & $0.20(0.17)$ & $0.53(0.15)$ & $0.08(0.11)$ \\
\hline Words encoded with distractors & $0.84(0.14)$ & $0.20(0.17)$ & $0.52(0.12)$ & $0.08(0.11)$ \\
\hline \multicolumn{5}{|l|}{ Divided Attention } \\
\hline Words encoded with targets & $0.73(0.15)$ & $0.25(0.23)$ & $0.60(0.22)$ & $0.16(0.18)$ \\
\hline Words encoded with distractors & $0.75(0.16)$ & $0.25(0.23)$ & $0.47(0.16)$ & $0.16(0.18)$ \\
\hline
\end{tabular}

Note. LF-OD: low-frequency orthographically distinct words; LF-OC: low-frequency orthographically common words

words. According to Hunt and Elliot (1980), in the context of mixed study lists, the visual irregularity of LF-OD words would induce participants to perform a more detailed analysis of their orthographic properties, which in turn offsets any additional processing advantage induced by the ABE. Interestingly, Kirchhoff, Schapiro, \& Buckner (2005) reported that the recognition of orthographically distinctive words activated occipital and parietal regions more than did orthographically common words, and Swallow, Makovski and Jiang (2012) found that the detection of auditory targets enhanced activity in the primary visual cortex. This partial overlap in the neural underpinnings of the $\mathrm{ABE}$ and the ODE may potentially account for the observed redundancy in their memory consequences. More generally, our findings point to the conclusion that, in the recognition advantage of LF words, the ODE and the ABE share a common locus of action which primarily involves early-phase, obligatory processes of stimulus identification and comprehension, as opposed to latephase processes of controlled rehearsal and semantic elaboration (Criss \& Malmberg, 2008; Malmberg \& Nelson, 2003). In agreement, in previous studies all of these effects were found to be robust at short study time durations and their magnitude did not increase with longer study times (Mulligan \& Spataro, in press; Malmberg \& Nelson, 2003; Gouden \& Nicolas, 2012a). This is not to say, however, that the orthographic distinctiveness of words is the critical factor that drives the $\mathrm{ABE}$, nor that the advantage of low-frequency words is solely determined by their greater orthographic distinctiveness (Malmberg et al., 2002). The current analysis suggests that other variables whose effects are primarily exerted in the early phases of memory encoding might also modulate the ABE. Future studies could address this issue, by looking at other secondary distinctiveness effects, like semantic isolation (Smith \& Hunt, 2000), which are more reliant on late-phase encoding processes.

Besides supporting the early-phase-elevated account of the $\mathrm{ABE}$, the present study provided important evidence about the attentional requirements underlying the ODE. One of the simplest explanations of this effect is based on the assumption that the elaboration of distinct items require increased attention at encoding (Hunt \& Elliott, 1980). Although previous studies have attempted to test the attentional hypothesis of the ODE by either using dual task paradigms or examining the performance of older adults, the conclusions have been mixed. Geraci and Rajaram (2002) reported that divided attention at encoding reduced the ODE in free recall and completely eliminated it in word-fragment cued recall, and suggested that the effect involves high-level comparative processing of common and distinct words. However, a later research by Gounden and Nicolas (2012b) found that the ODE was significant and equal in size in the FA and DA conditions. This same study showed that the ODE was not reduced in a sample of older adults (who presumably have reduced attentional resources), leading the authors to conclude that the effect reflects automatic perceptual processing. The present findings are more in line with the account proposed by Geraci and Rajaram (2002), because the ODE was eliminated in the DA condition and the request to monitor and respond to primarytask targets reduced the recognition of co-occurring LF-OD but not LF-OC, words. An important difference with respect to previous experiments is that our secondary task was presented in the visual modality, whereas both Geraci and Rajaram (2002) and Gounden and Nicolas (2012b) employed auditory digit-monitoring tasks. In light of past evidence indicating that the ODE is by and large a visual phenomenon-the effect does not occur when words are presented aurally (Hunt \& Elliott, 1980) - it seems reasonable to propose that our oddball task diverted modality-specific resources from encoding the visual properties of LF-OD words. Additional studies are needed to test whether the disruption of the ODE can be prevented by using an auditory oddball task (Swallow \& Jiang, 2010).

In summary, the present experiment showed that the $\mathrm{ABE}$ enhanced the recognition of LD-OC words, but had no effect on LF-OD words, when using a mixed-list design. This finding confirms and extends previous results suggesting that the 
ABE does not enhance encoding and memory of stimuli that are already subject to heightened attention at encoding (Mulligan et al., 2014), and further supports the early-phaseelevated-attention hypothesis of the ABE (Mulligan \& Spataro, in press).

Acknowledgments The authors would like to thank Stephen Goldinger, Kenneth J. Malmberg and an anonymous reviewer for their helpful suggestions on a previous version of the manuscript.

\section{References}

Criss, A. H., \& Malmberg, K. J. (2008). Evidence in favor of the earlyphase elevated-attention hypothesis: The effects of letter frequency and object frequency. Journal of Memory and Language, 59, 331345.

Geraci, L., \& Rajaram, S. (2002). The orthographic distinctiveness effect on direct and indirect tests of memory: Delineating the awareness and processing requirements. Journal of Memory and Language, 47, 273-291.

Gounden, Y., \& Nicolas, S. (2012a). The impact of processing time on the bizarreness and orthographic distinctiveness effects. Scandinavian Journal of Psychology, 53, 287-294.

Gounden, Y., \& Nicolas, S. (2012b). Ageing and secondarydistinctiveness-based effects: The orthographic distinctiveness effect is more robust than the bizarreness effect. The Quarterly Journal of Experimental Psychology, 65, 1820-1832.

Guttentag, R. E., \& Carroll, D. (1997). Recollection-based recognition: Word frequency effects. Journal of Memory and Language, 37, 502-516.

Hirshman, E., Trembath, D., \& Mulligan, N. (1994). Theoretical implications of the mnemonic benefits of perceptual interference. Journal of Experimental Psychology: Learning, Memory, and Cognition, 20, 608-620.

Hirshman, E., \& Palij, M. (1992). Rehearsal and the word frequency effect in recognition memory. Journal of Memory and Language, 31(4), 477-484. doi:10.1016/0749-596X(92)90024-R

Hunt, R., \& Elliot, J. (1980). The role of nonsemantic information in memory: Orthographic distinctiveness effects on retention. Journal of Experimental Psychology: General, 109, 49-74.

Kirchhoff, B. A., Schapiro, M. L., \& Buckner, R. L. (2005). Orthographic Distinctiveness and Semantic Elaboration Provide Separate
Contributions to Memory. Journal of Cognitive Neuroscience, 17, 1841-1854.

MacLeod, C. M., \& Kampe, K. E. (1996). Word frequency effects on recall, recognition, and word fragment completion tests. Journal of Experimental Psychology: Learning, Memory, and Cognition, 22, $132-142$.

Malmberg, K. J., \& Nelson, T. O. (2003). The word frequency effect for recognition memory and the elevated-attention hypothesis. Memory \& Cognition, 31, 35-43.

Malmberg, K. J., Steyvers, M., Stephens, J. D., \& Shiffrin, R. M. (2002). Feature frequency effects in recognition memory. Memory \& Cognition, 30, 607-613.

MacLeod, C. M., \& Masson, M. J. (1997). Priming patterns are different in masked word identification and word fragment completion. Journal of Memory And Language, 36, 461-483.

Mulligan, N. W. (2008). Attention and Memory. In H. L. Roediger (Ed.), Learning and Memory: A Comprehensive Reference (pp. 7-22). Oxford: Elsevier

Mulligan, N. W., \& Spataro, P. (in press). Divided Attention Can Enhance Early-Phase Memory Encoding: The Attentional Boost Effect and Study Trial Duration. Journal of Experimental Psychology: Learning, Memory, and Cognition.

Mulligan, N. W., Spataro, P., \& Picklesimer, M. (2014). The Attentional Boost Effect With Verbal Materials. Journal of Experimental Psychology: Learning, Memory, and Cognition, 40, 1049-1063.

Smith, R. E., \& Hunt, R. (2000). The effects of distinctiveness require reinstatement of organization: The importance of intentional memory instructions. Journal of Memory and Language, 43, 431-446.

Spataro, P., Cestari, V., \& Rossi-Arnaud, C. (2011). The Relationship between Divided Attention and Implicit Memory: A Meta-Analysis. Acta Psychologica, 136, 329-339.

Spataro, P., Mulligan, N. W., \& Rossi-Arnaud, C. (2013). Divided attention can enhance memory encoding: The attentional boost effect in implicit memory. Journal of Experimental Psychology: Learning, Memory, and Cognition, 39, 1223-1231.

Swallow, K. M., \& Jiang, Y. V. (2010). The attentional boost effect: Transient increases in attention to one task enhance performance in a second task. Cognition, 115, 118-132.

Swallow, K. M., Makovski, T., \& Jiang, Y. V. (2012). The selection of events in time enhances activity throughout early visual cortex. Journal of Neurophysiology, 108, 3239-3252.

Swallow, K. M., \& Jiang, Y. V. (2013). Attentional load and attentional boost: A review of data and theory. Frontiers in Psychology, 4. 